\title{
POLARIZATION OF TAU LEPTONS PRODUCED IN QUASIELASTIC NEUTRINO-NUCLEON SCATTERING
}

\author{
Konstantin S. Kuzmin, ${ }^{1,2}$ Vladimir V. Lyubushkin ${ }^{1,3}$ and Vadim A. Naumov ${ }^{1,4}$ \\ (1) Joint Institute for Nuclear Research, Dubna, Russia \\ (2) Institute for Theoretical and Experimental Physics, Moscow, Russia \\ (3) Physics Department of Irkutsk State University, Irkutsk, Russia \\ (4) Dipartimento di Fisica and INFN, Sezione di Firenze, Sesto Fiorentino, Italy
}

\begin{abstract}
A numerical analysis of the polarization vector of $\tau$ 's produced through quasielastic $\nu_{\tau}$ and $\bar{\nu}_{\tau}$ interactions with nucleons is given with two models for vector electromagnetic form factors of proton and neutron. The impact of $G$ parity violating axial and vector second-class currents is investigated by applying a simple heuristic model for the induced scalar and tensor form factors.
\end{abstract}

\section{Introduction}

The success of many current and future experimental projects for exploring neutrino oscillations and decay, nonstandard neutrino interactions, baryon number nonconservation and related phenomena revolves around the unambiguous reconstruction of $\tau$ lepton events generated in neutrino-matter interactions and detected through the secondary particles produced in the $\tau$ decays. For instance, the $\tau$ lepton events provide a clear signature of $\nu_{\mu}-\nu_{\tau}$ mixing; besides they are a source of unavoidable background to the proton decay and $n \bar{n}$ transition experiments. The momentum configuration of the $\tau$ decay secondaries is a functional of the $\tau$ lepton polarization; the latter is therefore a substantial input parameter for the data processing in the mentioned experiments.

In recent paper by Hagiwara et al. [1] the exact formulas for the polarization density matrix of a lepton produced in $\nu N$ and $\bar{\nu} N$ interactions have been derived within a noncovariant approach and under the Standard Model assumptions. This result has been generalized in our work [2] within a covariant method and by taking into account the nonstandard contributions induced by vector and axial second-class currents (SCC) [3-6]. Since the Standard Model contains the first-class currents (FCC) only, it is instructive to examine possible manifestations of the SCC induced interactions and grasp the uncertainties due to SCC in the future accelerator and astrophysical neutrino experiments. In this letter, by applying the relevant results of Ref. [2], we briefly discuss some consequences of the SCC induced contributions potentially measurable in future experiments on quasielastic (QE) $\nu_{\tau}$ and $\bar{\nu}_{\tau}$ interactions with nucleons.

\section{FCC effects}

The hadronic currents describing the $\Delta Y=0$ processes $\nu_{\tau} n \rightarrow \tau^{-} p$ and $\bar{\nu}_{\tau} p \rightarrow \tau^{+} n$ may be written as [3]

$$
\left\langle p, p^{\prime}\left|\widehat{J}_{\alpha}^{+}\right| n, p\right\rangle=\cos \theta_{C} \bar{u}_{p}\left(p^{\prime}\right) \Gamma_{\alpha} u_{n}(p), \quad\left\langle n, p^{\prime}\left|\widehat{J}_{\alpha}^{-}\right| p, p\right\rangle=\cos \theta_{C} \bar{u}_{n}\left(p^{\prime}\right) \bar{\Gamma}_{\alpha} u_{p}(p),
$$

where $\theta_{C}$ is the Cabibbo angle and the vertex function

$$
\Gamma_{\alpha}=\gamma_{\alpha} F_{V}+i \sigma_{\alpha \beta} \frac{q_{\beta}}{2 M} F_{M}+\frac{q_{\alpha}}{M} F_{S}+\left(\gamma_{\alpha} F_{A}+\frac{p_{\alpha}+p_{\alpha}^{\prime}}{M} F_{T}+\frac{q_{\alpha}}{M} F_{P}\right) \gamma_{5} .
$$

is defined through the six, in general complex, form factors $F_{i}\left(q^{2}\right)$ : FCC induced $(i=V, M, A, P$ ) and SCC induced $(i=S, T)$. Here $p$ and $p^{\prime}$ are, respectively, the 4-momenta of the initial and final nucleons, $q=p^{\prime}-p$ is the 4-momentum transfer and $M$ is the nucleon mass (as usually, the half-sum of proton and neutron masses). Hereafter we follow the notation of Ref. [2]. 
We investigate two models for the Sachs form factors of proton and neutron: the "benchmark" dipole model [3] with the standard value of vector mass $\left(M_{V}=0.84 \mathrm{GeV} / c^{2}\right)$ and the GariKrüempelmann (GK) model [7] extended and fine-tuned by Lomon [8] to match current experimental data. Specifically we explore the so-called "GKex(02S)" model which fits the modern and consistent older data well and meets the requirements of dispersion relations and of QCD at low and high 4-momentum transfer.

Note that the GKex(02S) model is very close numerically the "BBA-2003" parametrization by Budd et al. [9] obtained through a global fit to the data from Rosenbluth analysis of elastic ep cross section measurements and those from the polarization transfer techniques. Both these models do not take into account the two-photon contribution corrections to the form factors (see Ref. [10] and references therein) as well as the most recent data from JLab and MAMI (which are however in good agreement with the GKex(02S) and BBA-2003).

For the axial and pseudoscalar form factors we use the conventional parametrizations

$$
F_{A}\left(q^{2}\right)=F_{A}(0)\left(1-\frac{q^{2}}{M_{A}^{2}}\right)^{-2} \quad \text { and } \quad F_{P}\left(q^{2}\right)=\frac{2 M^{2}}{m_{\pi}^{2}-q^{2}} F_{A}\left(q^{2}\right)
$$

with $F_{A}(0)=g_{A}=-1.2695 \pm 0.0029$. The presently available experimental data on the axial mass, $M_{A}$, show very wide spread, from roughly 0.6 to $1.2 \mathrm{GeV} / c^{2}$ [11] with the weighted average of $1.026 \pm 0.021 \mathrm{GeV} / c^{2}$. Side by side with the pseudoscalar form factor, $F_{P}\left(q^{2}\right)$, this is the main source of uncertainties for predicting the $\nu_{\tau} n$ and $\bar{\nu}_{\tau} p \mathrm{QE}$ cross sections and polarization effects. Since the pseudoscalar form factor contribution enters multiplied by $m_{\ell} / M$, it is small for the electron and muon production but substantial for $\tau$ production, especially for small $\left|q^{2}\right|$ (since $\left|F_{P}\right| \gg\left|F_{A}\right|$ for $\left.\left|q^{2}\right| \ll M^{2}\right)$. Let us remark that the "standard" expression for the pseudoscalar form factor is only a (doubtful) parametrization inspired by the PCAC hypothesis and the assumption that the pion pole dominates at $\left|q^{2}\right| \lesssim m_{\pi}^{2}$ [3]. A discussion of some other phenomenological models for the $F_{P}$ and their impact to the $\tau$ lepton production cross section and polarization is given recently by Hagiwara et al. [12]. For that reason we do not concern this important issue in present study.

For evaluating the lepton polarization vector $\mathcal{P}=\left(\mathcal{P}_{P}, \mathcal{P}_{T}, \mathcal{P}_{L}\right)$ we apply generic formulas given in Ref. [2]. In this section we will neglect the nonstandard contributions. The transversal polarization, $\mathcal{P}_{T}$, is therefore exactly zero. The longitudinal polarization, $\mathcal{P}_{L}$, perpendicular polarization, $\mathcal{P}_{P}$, and Lorentz invariant degree of polarization

$$
|\mathcal{P}|=\sqrt{\mathcal{P}_{L}^{2}+\mathcal{P}_{P}^{2}+\mathcal{P}_{T}^{2}}
$$

evaluated with the dipole and $\operatorname{GKex}(02 S)$ models for the vector electromagnetic form factors and with $M_{A}=1 \mathrm{GeV} / c^{2}$ are shown in figs. 1, 2] and 3 respectively, as functions of the lepton scattering angle $\theta$ at several (anti)neutrino energies (both variables are measured in the laboratory frame). Due to the large $\tau$ lepton mass, $m_{\tau}$, for each allowed pair of variables $\left(E_{\nu}, \theta\right)$ there are two solutions ("branches") of the energy-momentum conservation equations with respect to the lepton momentum value $P_{\tau}=\left|\mathbf{p}_{\tau}\right|$. These are given by

$$
P_{\tau}^{ \pm}(\theta)=\frac{E_{\nu}^{*}\left(M E_{\tau}^{*} \cos \theta \pm m_{\tau} E_{N}^{*} \sqrt{\zeta^{2}-\sin ^{2} \theta}\right)}{M^{2}+\left(E_{\nu}^{*}\right)^{2} \sin ^{2} \theta}, \quad \zeta=\frac{\left(E_{\nu}^{*}+E_{N}^{*}\right) P_{\tau}^{*}}{m_{\tau} E_{\nu}} .
$$

Here $P_{\tau}^{*}$ is the lepton momentum, $E_{\nu}^{*}, E_{\tau}^{*}$ and $E_{N}^{*}$ are the energies of (anti)neutrino, lepton and target nucleon, respectively, measured in the center-of-mass frame. The physically allowed interval of scattering angles is

$$
0 \leq \theta \leq \arcsin (\zeta) \leq \arcsin \left(M / m_{\tau}\right) \approx 31.9^{\circ}
$$

The main kinematic branch, $P_{\tau}^{+}(\theta)$, whose contribution into the total QE cross section is dominant, is shown in the figures by solid curves and the second branch, $P_{\tau}^{-}(\theta)$, is shown by dashed curves. The dotted curves display the boundary between the two branches which is defined by the condition $\sin \theta=\zeta$ [and thus $\left.P_{\tau}=2 m_{\tau}^{2} E_{\nu} \cos \theta /\left(2 M E_{\nu}+m_{\tau}^{2}\right)\right]$. 
First of all, as is seen from the figures, the behavior of the $\tau$ lepton polarization is quite different from the naively expected. At low energies and small scattering angles (the most important kinematic range for underground neutrino experiments), the degree of polarization of $\tau^{-}\left(\tau^{+}\right)$is far from unity (close to unity) for the main branch. There always exist the nontrivial perpendicular (to the reaction plane) polarization for both $\tau^{-}$and $\tau^{+}$but their dependencies from $E_{\nu}$ and $\theta$ are distinctly different for $\tau^{-}$and $\tau^{+}$. It is also clear that the components of the polarization vector as well as the degree of polarization of $\tau^{-}$are almost insensitive to the model (except for the near-threshold energies). This is in clear contrast to the case of $\tau^{+}$lepton, for which the polarization vector is very sensitive to the shapes of the vector electromagnetic form factors for essentially any antineutrino energy.

\section{SCC effects}

A major problem in the quantitative analysis of the SCC effects is the $q^{2}$ dependence of the induced scalar and tensor form factors. To get some feeling for how big the SCC effects could be, let us consider the following toy model for the form factors:

$$
\begin{aligned}
& F_{S}\left(q^{2}\right)=\xi_{S} e^{i \phi_{S}} F_{V}(0)\left(1-\frac{q^{2}}{M_{S}^{2}}\right)^{-2}, \\
& F_{T}\left(q^{2}\right)=\xi_{T} e^{i \phi_{T}} F_{A}(0)\left(1-\frac{q^{2}}{M_{T}^{2}}\right)^{-2} .
\end{aligned}
$$

The model includes six free parameters, $\xi_{S, T} \geq 0, \phi_{S, T}$ and $M_{S, T}$ and is an elementary amplification of the models adopted by experimental collaboration at FNAL [13], IHEP (Serpukhov) [14] and BNL $[15,16]$ to constrain the SCC strengths from the measurements of $\nu / \bar{\nu}$ interactions with nucleons. All the measurements [13-16] found no significant indication of nonzero SCC contributions.

The strongest $90 \%$ C.L. upper limit on the axial SCC strength $\xi_{T}$ has been obtained at the Brookhaven Alternating Gradient Synchrotron (BNL AGS) experiment with a wide-band $\bar{\nu}_{\mu}$ beam with $\left\langle E_{\bar{\nu}}\right\rangle=1.2 \mathrm{GeV},\left|q^{2}\right|<1.2(\mathrm{GeV} / c)^{2}$ [16] as a function of the "tensor mass" $M_{T}$, assuming CVC $\left(\xi_{S}=0\right)$ and the dipole model for the Sachs form factors of the proton. The limit ranges from about 0.78 at $M_{T}=0.5 \mathrm{GeV} / c^{2}$ to about 0.11 at $M_{T}=1.5 \mathrm{GeV} / c^{2}$. The BNL AGS constraint at the lower limit of $M_{T}$ is not too informative since it is about 7 times weaker than that follows from the nuclear structure studies [5] while at the upper limit it is appropriate for our heuristic exploration.

The BNL AGS constraint to the vector SCC (violating the standard CVC hypothesis) is not so robust $\left(\xi_{S}<1.8\right.$ at $M_{S}=1.0 \mathrm{GeV} / c^{2}$ and assuming $\left.\xi_{T}=0\right)$ and should not be taken too literally since the scalar form factor contribution to the QE muon production cross section is reduced by factor of $\left(m_{\mu} / M\right)^{2} \sim 0.01$. However this is the only, to our knowledge, experimental limit to the CVC violation obtained at comparatively large momentum transfer. The value of $M_{A}$ simultaneously obtained in the BNL AGS experiment was $1.09 \mathrm{GeV} / \mathrm{c}^{2}$ which is the same as the value obtained assuming no SCC.

Notice that there is no any reduction of the vector SCC contribution to the QE $\tau$ production $\left[\left(m_{\tau} / M\right)^{2} \approx 3.6\right]$ and hence even small violation of the CVC principle could affect both the $\tau$ lepton production cross section and polarization.

In figs. 4 and 5 we show a representative example for the ratios of the $\tau^{\mp}$ lepton degree of polarization, $|\mathcal{P}|$, evaluated with nonzero $\xi_{T, S}$ to those with $\xi_{T, S}=0$. Only the dipole model for the Sachs form factors is utilized here since the relative SCC effects are qualitatively the same for the GKex(02S) model. The calculations are done with $M_{A}=1.0 \mathrm{GeV} / c^{2}$ and $\phi_{T, S}=0$. The latter restriction is equivalent to the time reversal invariance while the nonzero strength parameters $\xi_{T, S}$ violate the charge symmetry of weak interactions.

Our choice of the unknown parameters $M_{T}$ and $\xi_{T}$ is made to ensure the BNL AGS limits [16] (very sensitive to $M_{T}$ ) as well as the restrictions on the axial SCC coupling constant from studies of $\beta$ decay of complex nuclei [5] (much less sensitive to $M_{T}$ ). As a conservative upper limit, we accept $\xi_{T}=0.1$ and $M_{T}=1.5 \mathrm{GeV} / c^{2}$. The values $M_{S}=1.0 \mathrm{GeV} / c^{2}$ and $\xi_{S}=1.8$ are taken according to the BNL AGS upper limit in order to demonstrate the maximum possible effect of the vector current nonconservation. 
As is seen from the figures, the impact of the tensor and (especially) scalar form factors is not negligible in the kinematic regions where the differential QE cross section $d \sigma / d\left|q^{2}\right|$ is comparatively large (the main kinematic branch, small scattering angles). On the other hand, the axial SCC contribution (which is not forbidden by any basic principle like CVC) is not too dramatic and, especially for $\tau^{+}$, the polarization vector is more sensitive to small variations of the standard axial and pseudoscalar form factors [12]. This is a handicap for experimental probe of the SCC effects by $\nu_{\tau}$ and $\bar{\nu}_{\tau}$. On the other hand, this is an advantage for the current and future neutrino oscillation experiments since the SCC contributions are not the main source of systematic uncertainties.

Let us emphasize that similar effects for the muon polarization vector are uninterestingly small; so we do not discuss the corresponding numerical examples here.

For the last and more speculative possibility, we consider effects of the phase factors $\exp \left(i \phi_{S, T}\right)$ violating the $T$ invariance. It can be shown that, for the allowed values of the strength parameter $\xi_{T}$, the lepton polarization vector is not significantly affected by the "tensor phase" $\phi_{T}$. However it is not in general the case for the "scalar phase" $\phi_{S}$. The effect is shown in fig. 6 for the sine of $\Psi$, the angle between the momentum and polarization vector of a $\tau^{-}\left(\tau^{+}\right)$lepton generated quasielastically by a $10 \mathrm{GeV} \nu_{\tau}\left(\bar{\nu}_{\tau}\right)$. The angle $\Psi$ is defined by

$$
\sin \Psi=\frac{\sqrt{\mathcal{P}_{P}^{2}+\mathcal{P}_{T}^{2}}}{|\mathcal{P}|} \quad \text { or } \quad \cos \Psi=\frac{\mathcal{P}_{L}}{|\mathcal{P}|} .
$$

Due to the nontrivial phase $\phi_{S}$, the transverse polarization, $\mathcal{P}_{T}$, is no longer exactly zero but remains comparatively small. One can prove that $\left|\mathcal{P}_{L}\right|,\left|\mathcal{P}_{P}\right|$ and $\left|\mathcal{P}_{T}\right|$ are invariant under the transformation $\phi_{S} \mapsto 2 \pi-\phi_{S}$. It is therefore sufficient to vary the scalar phase within the range $0^{\circ}$ to $180^{\circ}$. All the parameters used for this calculation are listed in the legends of fig. 6 The value $\xi_{S}=1.8$ is again taken to show the maximum possible effect allowed by the BNL AGS limit. As one can see from the figure, the vector SCC induced $C$ violating effects may be rather large. Figure 6 also demonstrates quite sizable transformation of $\sin \Psi$ when the phase $\phi_{S}$ varies from $0^{\circ}$ to $180^{\circ}$. For the main kinematic branch (solid lines), the $\sin \Psi$ is a decreasing function of the phase $\phi_{S}$ for $\tau^{-}$at almost any scattering angle. It is not the case for $\tau^{+}$: the $\sin \Psi$ is an increasing function of $\phi_{S}$ at $2^{\circ} \lesssim \theta \lesssim 18^{\circ}$ and rather nonmonotonic function for other allowed ranges of the scattering angle.

We recall in conclusion that our analysis is only valid within the adopted ad hoc model for the SCC induced form factors, including the quite arbitrary choice for the tensor and scalar mass values.

\section{Acknowledgments}

We thank S. M. Bilenky, A. V. Efremov, B. Z. Kopeliovich, D. V. Naumov, J. Soffer and O. V. Teryaev for useful conversations. V. L. and K. K. are grateful to the Physics Department of Florence University for warm hospitality and financial support of this work.

\section{References}

[1] K. Hagiwara, K. Mawatari and H. Yokoya, Nucl. Phys. B 668, 364 (2003) |hep-ph/0305324|.

[2] K. S. Kuzmin, V. V. Lyubushkin and V. A. Naumov, in Proc. of the 10th International Workshop on High-Energy Spin Physics (“SPIN’03”), Dubna, Russia, September 16-20, 2003 |hep-ph/0312107|.

[3] C. H. Llewellyn Smith, Phys. Rept. 3C, 261 (1972).

[4] L. Grenacs, Ann. Rev. Nucl. Part. Sci. 35, 455 (1985).

[5] D. H. Wilkinson, Eur. Phys. J. A7, 307 (2000); Nucl. Instrum. Meth. A455, 656 (2000); ibid. A469, 286 (2001).

[6] S. Gardner and C. Zhang, Phys. Rev. Lett. 86, 5666 (2001) |hep-ph/0012098|. 
[7] M. F. Gari and W. Krüempelmann, Phys. Lett. B274, 159 (1992); erratum - ibid. B282, 483 (1992).

[8] E. L. Lomon, Phys. Rev. C66, 045501 (2002) [nucl-th/0203081].

[9] H. Budd, A. Bodek and J. Arrington, hep-ex/0308005

[10] J. Arrington, hep-ph/0408261

[11] V. Bernard, L. Elouadrhiri and Ulf-G. Meißner, J. Phys. G28, R1 (2002) |hep-ph/0107088|.

[12] K. Hagiwara, K. Mawatari and H. Yokoya, Phys. Lett. B591, 113 (2004) |hep-ph/0403076].

[13] J. Bell et al., Phys. Rev. Lett. 40, 1226 (1978).

[14] S. V. Belikov et al., Z. Phys. A320, 625 (1985).

[15] N. J. Baker et al., Phys. Rev. D23, 2499 (1981); see also M. J. Murtagh, in Proc. of the International Conference on Neutrino Physics and Astrophysics, "Neutrino'81", Wailea, Hawaii, July 1-8, 1981, eds. R. J. Cence et al., Vol. 2, p. 388 and preprint BNL-30082 (1981).

[16] L. A. Ahrens et al., Phys. Lett. B202, 284 (1988). 


\section{FIGURES}
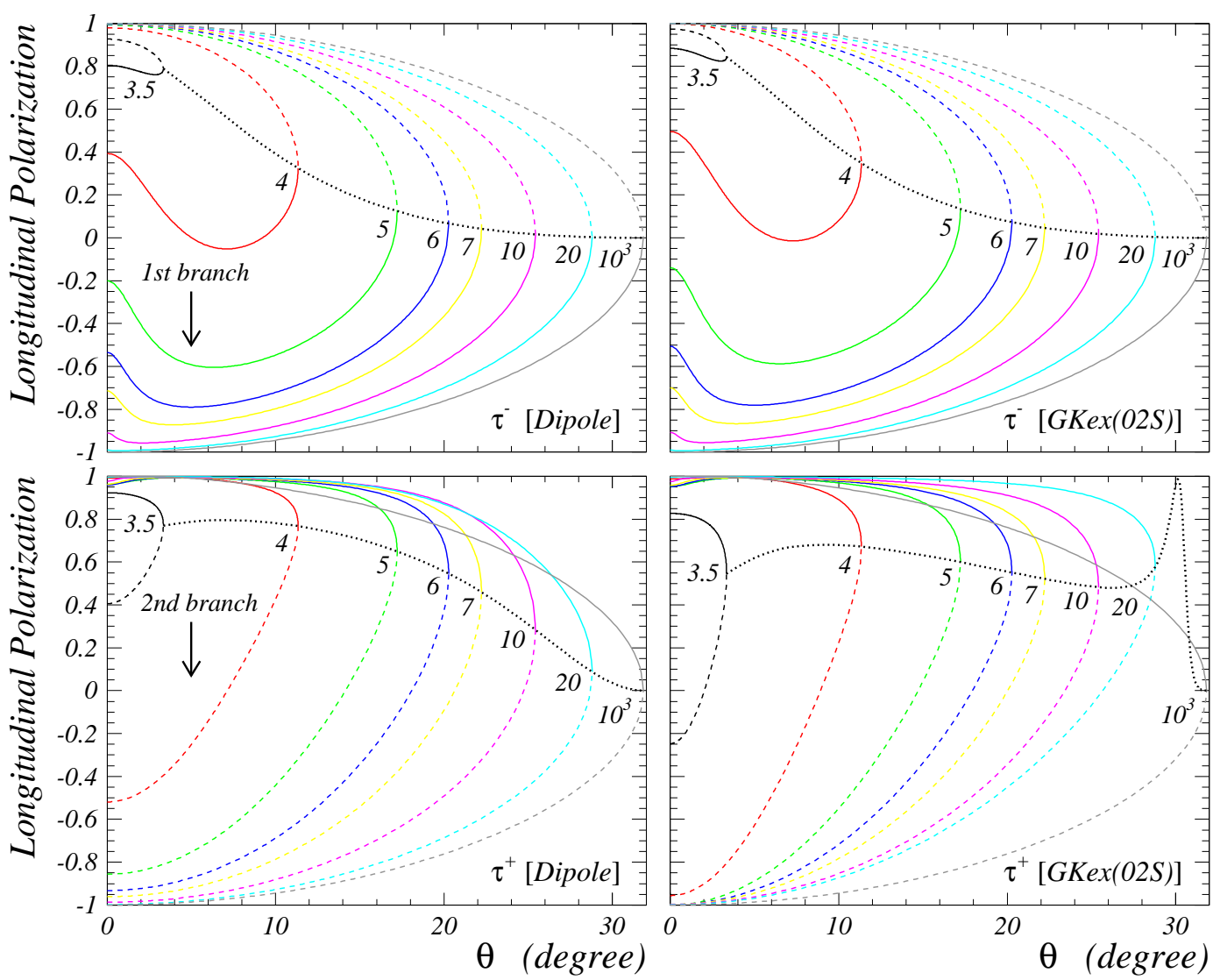

Figure 1: Longitudinal polarization, $\mathcal{P}_{L}$, evaluated with the dipole and $\operatorname{GKex}(02 \mathrm{~S})$ models for the nucleon electromagnetic form factors at different (anti)neutrino energies (shown near the curves in $\mathrm{GeV}$ ). The gray dotted curves indicate the boundaries between the two kinematically allowed solutions. The main kinematic branches are shown by solid curves. 

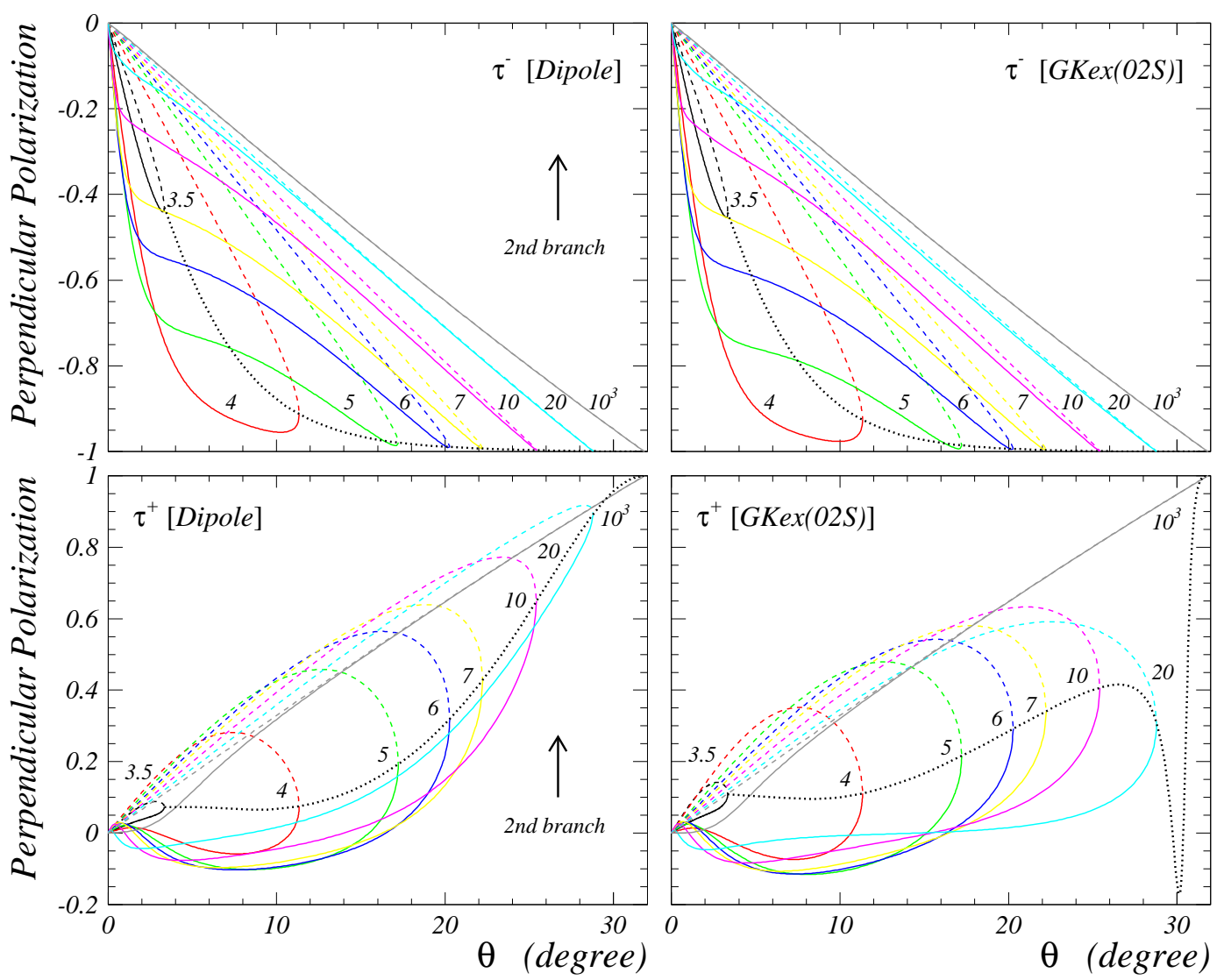

Figure 2: Perpendicular polarization, $\mathcal{P}_{P}$, evaluated with the dipole and GKex $(02 \mathrm{~S})$ models for the nucleon electromagnetic form factors at different (anti)neutrino energies (shown near the curves in $\mathrm{GeV}$ ). The gray dotted curves indicate the boundaries between the two kinematically allowed solutions. The main kinematic branches are shown by solid curves. 

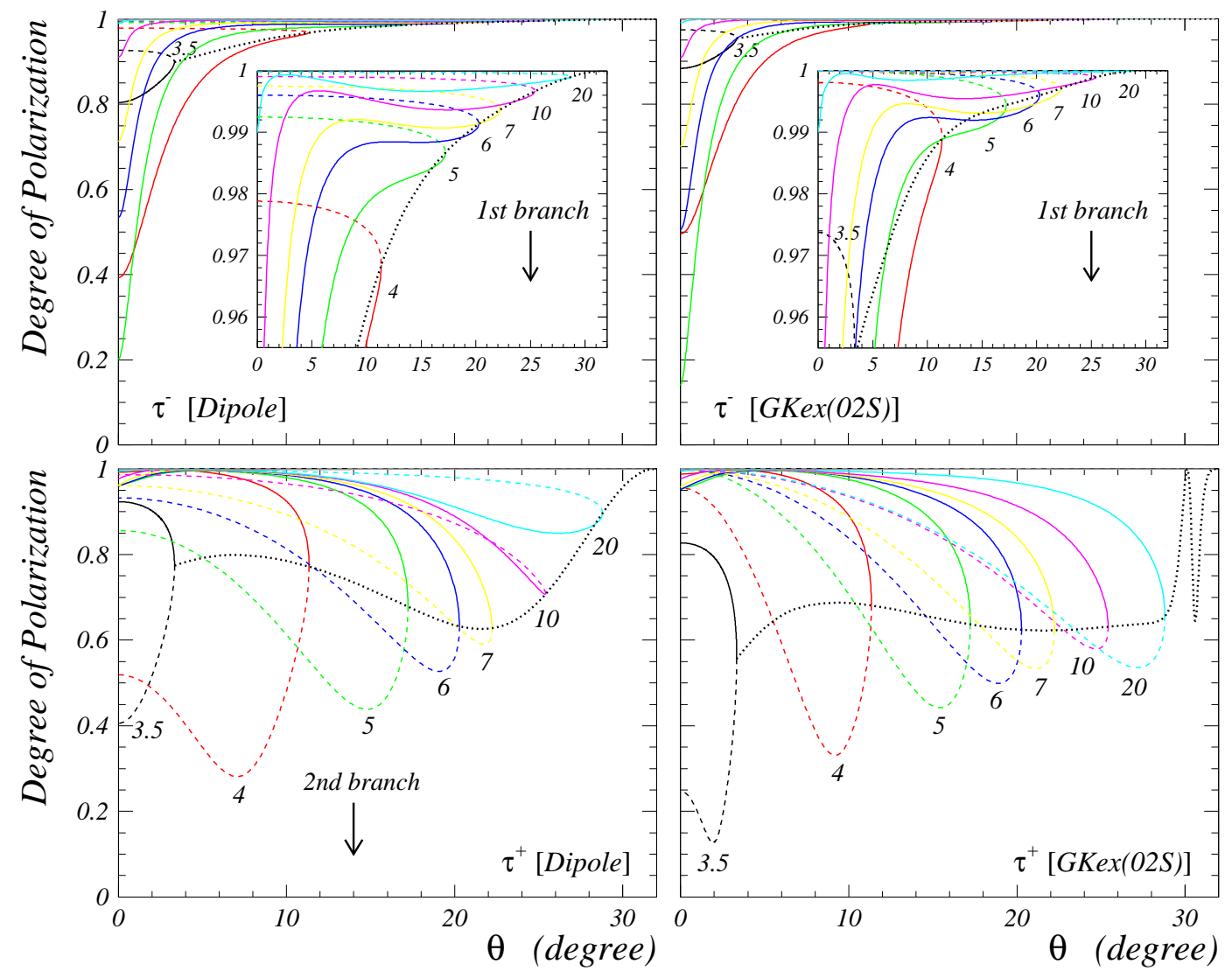

Figure 3: Degree of polarization, $|\mathcal{P}|$, evaluated with the dipole and $\operatorname{GKex}(02 \mathrm{~S})$ models for the nucleon electromagnetic form factors at different (anti)neutrino energies (shown near the curves in $\mathrm{GeV}$ ). The gray dotted curves indicate the boundaries between the two kinematically allowed solutions. The main kinematic branches are shown by solid curves. The insets in two upper panels show a zoomed view. 

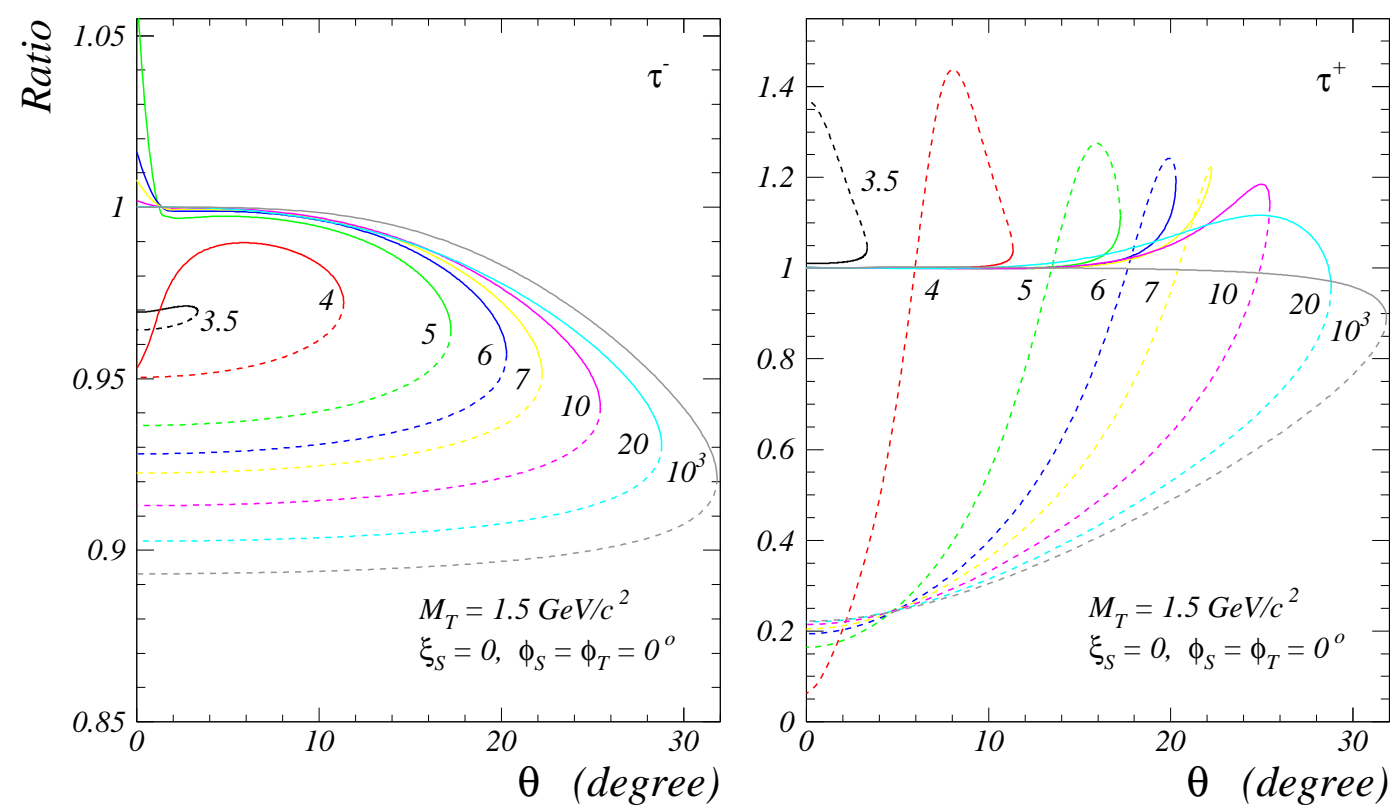

Figure 4: Ratios $\left|\mathcal{P}\left(\xi_{T}=0.1\right)\right| /\left|\mathcal{P}\left(\xi_{T}=0\right)\right|$ evaluated with the dipole model for the nucleon electromagnetic form factors and with $M_{A}=1.0 \mathrm{GeV} / c^{2}$. Values of the SCC parameters are given by the legends; (anti)neutrino energies are shown near the curves in GeV. The main kinematic branches are shown by solid curves.
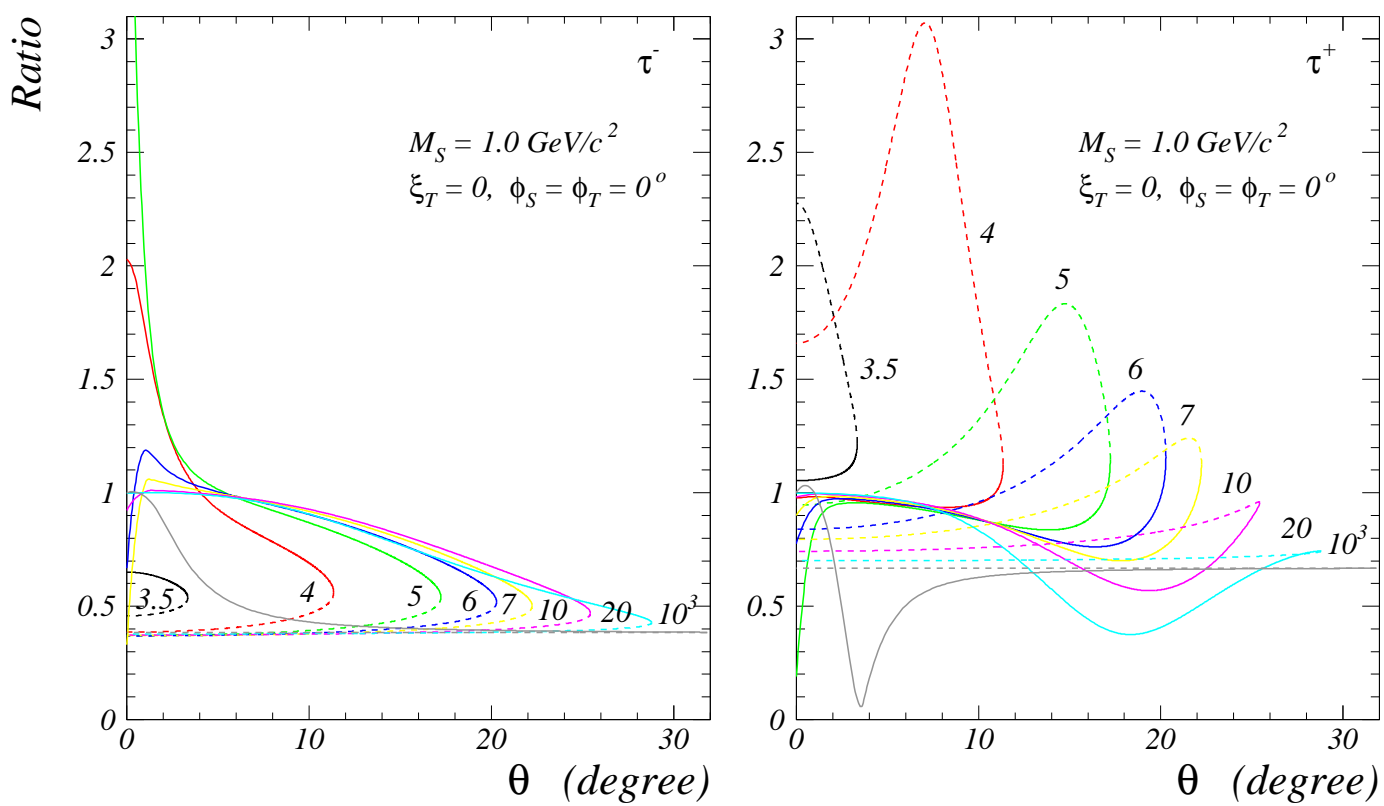

Figure 5: Ratios $\left|\mathcal{P}\left(\xi_{S}=1.8\right)\right| /\left|\mathcal{P}\left(\xi_{S}=0\right)\right|$ evaluated with the dipole model for the nucleon electromagnetic form factors and with $M_{A}=1.0 \mathrm{GeV} / c^{2}$. Values of the SCC parameters are given by the legends; (anti)neutrino energies are shown near the curves in $\mathrm{GeV}$. The main kinematic branches are shown by solid curves. 

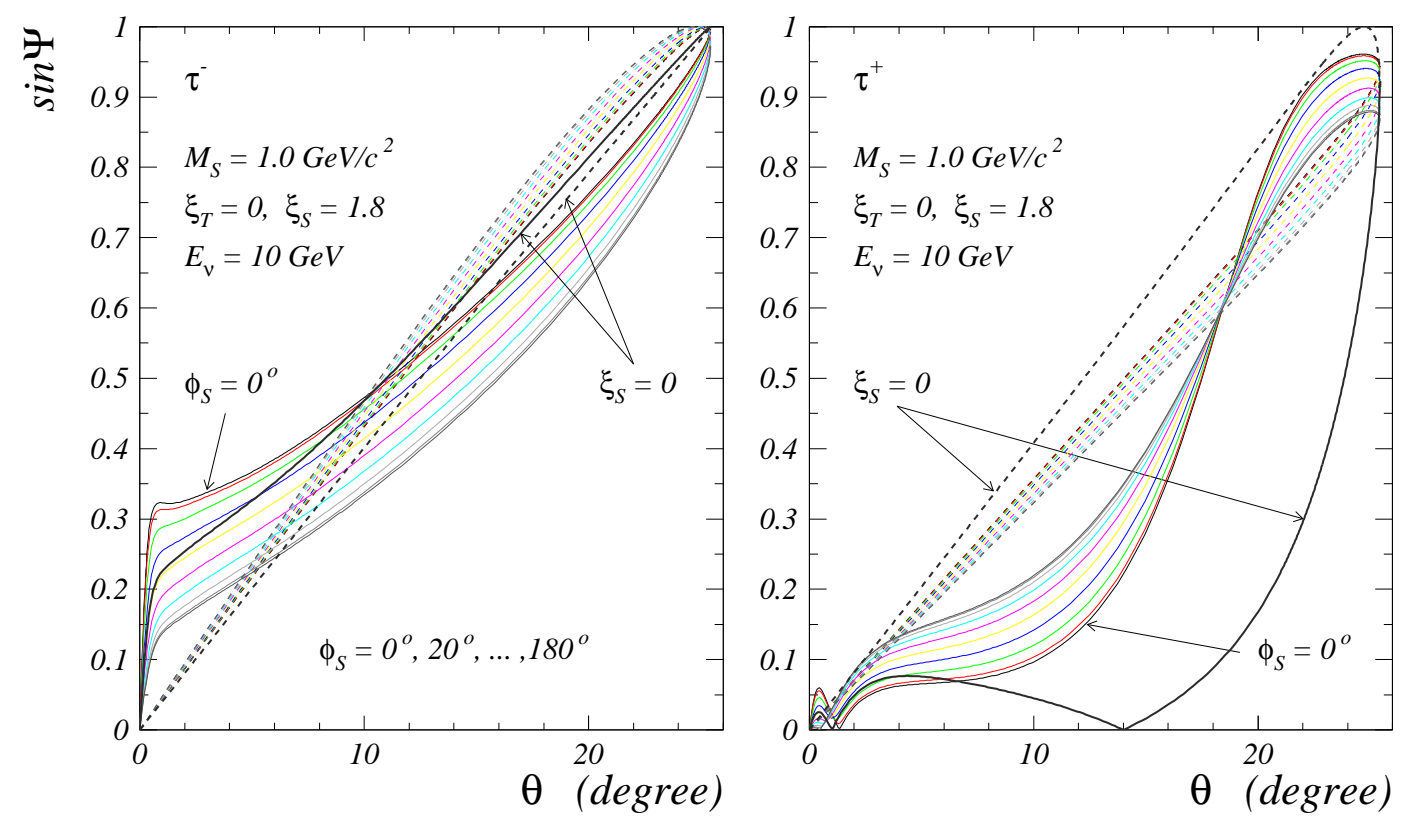

Figure 6: $\sin \Psi$ as a function of scattering angle $\theta$ at (anti)neutrino energy $E_{\nu}=10 \mathrm{GeV}$ for different values of the "scalar phase" $\phi_{S}$ (from $0^{\circ}$ to $180^{\circ}$ with step of $20^{\circ}$ ). The calculations are done with the dipole model for the nucleon electromagnetic form factors and with $M_{A}=1.0 \mathrm{GeV} / c^{2}$. Values of the remaining SCC parameters are given by the legends. The main kinematic branches are shown by solid curves. The case $\xi_{S}=0$ is also shown by thick gray curves. 